\title{
Ultrafast collective oxygen-vacancy flow in Ca-doped $\mathrm{BiFeO}_{3}$
}

\author{
Ji Soo Lim ${ }^{1,2}$, Jin Hong Lee $\mathbb{B}^{1,2}$, Heung-Sik Park ${ }^{1,2}$, Ran Gao ${ }^{3}$, Tae Yeong Koo ${ }^{4}$, Lane W. Martin $\mathbb{B}^{3,5}$, \\ Ramamoorthy Ramesh ${ }^{3,5,6}$ and Chan-Ho Yang ${ }^{1,2,7}$
}

\begin{abstract}
The ultrafast motion of oxygen vacancies in solids is crucial for various future applications, such as oxide electrolytes. Visualization and quantification can offer unforeseen opportunities to probe the collective dynamics of defects in crystalline solids, but little research has been conducted on oxygen vacancy electromigration using these approaches. Here, we visualize electric-field-induced creation and propagation of oxygen-vacancy-rich and -poor competing phases and their interface with optical contrast in Ca-substituted $\mathrm{BiFeO}_{3}$ that contains a high density of mobile oxygen vacancies. We quantitatively determined the drift velocity of collective migration to be on the order of $100 \mathrm{\mu m} \mathrm{s}^{-1}$ with an activation barrier of $0.79 \mathrm{eV}$, indicating a significantly large ionic mobility of $2 \times 10^{-6} \mathrm{~cm}^{2} \mathrm{~s}^{-1} \mathrm{~V}^{-1}$ at a remarkably low temperature of $390^{\circ} \mathrm{C}$. In addition, visualization enables direct observation of fluidic behavior, such as the enhancement of conduction at channel edges, which results in U-shaped viscous propagation of the phase boundary and turbulence under a reverse electric field. All of these results provide new insights into the collective motion of defects.
\end{abstract}

\section{Introduction}

Ionized oxygen vacancies act as doubly charged electron donors in oxides and affect a variety of physical properties, such as superconductivity ${ }^{1}$, magnetoresistance ${ }^{2}$, and ferroelectricity ${ }^{3,4}$. Furthermore, a vast amount of research interest has been devoted to technological applications, including resistive switching memory ${ }^{5}$, solid oxide fuel cells (SOFCs) ${ }^{6}$, batteries ${ }^{7}$, sensors ${ }^{8}$, memresistors ${ }^{9}$, and neuromorphic computation ${ }^{10}$, that use the mobility of these species. In general, ionic conduction relies on ions hopping through an otherwise rigid crystal, and although multiple vacancies likely interact with each other at the high-density limit of the defect concentration, studies on the dynamics of ionic conductivity have primarily focused on single particles ${ }^{11}$. Thus, the understanding of collective

\footnotetext{
Correspondence: Chan-Ho Yang (chyang@kaist.ac.kr)

'Department of Physics, Korea Advanced Institute of Science and Technology (KAIST), Yuseong-gu, Daejeon 34141, Republic of Korea

${ }^{2}$ Center for Lattice Defectronics, KAIST, Yuseong-gu, Daejeon 34141, Republic of Korea
}

Full list of author information is available at the end of the article. behavior is lacking, which is especially troublesome considering that a nearby oxygen vacancy can give rise to considerable lattice deformation and electrostatic interactions and can significantly modify the activation barrier for ionic hopping ${ }^{12}$. At the same time, structural, electronic, and optical states are strongly influenced by the defect concentration. Thus, defect formation and migration in solids is a highly complex mutual coupling phenomenon that influences effects ranging from structural and electronic phase transitions to diffusional processes under inhomogeneous external/internal electric and strain fields and chemical pressures ${ }^{13-16}$. To pave the way for understanding this sophisticated dynamic phenomenon, it is necessary to better visualize the spatial distribution of the oxygen-vacancy concentration and observe the real-time evolution of the distribution in electric fields.

Ca-substituted $\mathrm{BiFeO}_{3}\left(\mathrm{Bi}_{1-x} \mathrm{Ca}_{x} \mathrm{FeO}_{3-\delta} ; \mathrm{BCFO}\right)$ is an ideal material for examining oxygen-vacancy dynamics since the aliovalent substitution of $\mathrm{Ca}^{2+}$ ions spontaneously creates oxygen vacancies directly proportional to

\section{(c) The Author(s) 2018}


the Ca content, i.e., $\delta=x / 2$ (e.g., $n \approx 2.49 \times 10^{21} \mathrm{~cm}^{-3}$ at $x=0.3$ ), to maintain the valence state of $\mathrm{Fe}^{3+}$ (ref. ${ }^{17}$ ). Such oxygen-deficient states are thermodynamically stable, and it has been reported that the defect concentration does not change significantly with thermal annealing under oxidizing conditions (100 atm), even at $800{ }^{\circ} \mathrm{C}$ (ref. ${ }^{18}$ ). Nevertheless, this material appears to be an excellent ionic conductor. Application of an external electric field at elevated temperatures spatially redistributes oxygen vacancies and produces hole carriers in the regions with lower oxygen-vacancy concentrations, resulting in an insulator-to-conductor transition and $p-n$ diode-like features in electronic conduction properties. Another advantage of this material is that virtually all oxygen vacancies can be transferred by electromigration, as confirmed by stoichiometry analysis via oxygen $1 s \mathrm{X}$ ray photoelectron spectroscopy ${ }^{19}$. Most importantly, this material has a strong color change induced by controlling the oxygen-vacancy concentration via the electrochromic effect $^{20}$. An optical transition from yellow to dark gray or black occurs with a decrease in the oxygen-vacancy content, allowing real-time observation of defect dynamics. Furthermore, as the parent compound is ferroelectric, the system has a large lattice instability, and thus, a defect induces significant local deformation in the lattice ${ }^{21,22}$. At the same time, the high bismuth ionic polarizability, which is related to the presence of $6 s$ lone-pair electrons, has been found to be one of the keys to promoting the migration of oxygen vacancies ${ }^{23,24}$. Ultimately, the compound, which contains inexpensive, abundant elements $(\mathrm{Bi}, \mathrm{Ca}$, and $\mathrm{Fe})$, is likely to be a promising ionic conductor; however, the oxygen-vacancy dynamics of this compound are not well known.

On this basis, we explored the visualization and quantification of ionic migration through a BCFO channel between two coplanar electrodes by spatial and temporal tracking of the local optical-color change. A significant advance was made by introducing a capping layer to protect the channel from reacting with the atmosphere, thereby leading to the realization of a quasiclosed oxygen-vacancy system. In this study, we report the collective behaviors of high-mobility oxygen vacancies in BCFO with $x=0.3$. Quantification of the vacancy evolution allows for the direct determination of kinematic variables such as the ionic-drift velocity and mobility and the thermodynamic activation barrier and diffusivity. Synchronized crystal structural analysis and channel current monitoring provide useful insights into the structural/electronic-phase evolution as a function of the defect concentration. Furthermore, the electrophoretic display ${ }^{25}$ offers versatile information concerning fluidic-like defect motion, such as turbulence, beyond the quantification of the kinematic/thermodynamic variables.

\section{Real-time visualization of electromigration}

An optical microscope equipped with a CCD camera is a simple yet powerful tool to visualize and trace oxygenvacancy migration through the 400- $\mu \mathrm{m}$-long BCFO channel (Fig. 1a). An optically transparent epitaxial $\mathrm{LaAlO}_{3}(10 \mathrm{~nm}$ in thickness) film is deposited on top of the BCFO channel. $\mathrm{LaAlO}_{3}$ acts as a capping layer to prevent the $\mathrm{BCFO}$ film from reacting with ambient oxygen because it has a higher oxygen-vacancy-formation energy of $\sim 7.3 \mathrm{eV}$ compared to that of other oxide materials $^{26,27}$. The BCFO films grew in a step-flow mode and show an atomically flat surface with a step-terrace structure, but the $\mathrm{LaAlO}_{3}$ capping layer was deposited in an island-growth mode (Supplementary Fig. 1). Applying an external DC voltage $(25 \mathrm{~V})$ at an elevated temperature causes the oxygen vacancies in the channel to migrate toward the negatively biased electrode. Since the channel size is finite, oxygen vacancies cannot be continuously supplied from the positive electrode side under a DC electric field. Eventually, a fully oxidized, stoichiometric phase arising from the removal of oxygen vacancies nucleates near the electrode. This region can be easily distinguished from the semitransparent, yellowish, asgrown insulating state because the newly formed phase is optically dark and electronically conducting due to the introduction of hole carriers as a result of the uncompensated Ca-substitution effect. The dark-contrast region expands toward the electric-field direction (Fig. 1b).

A video of the optical-contrast evolution was taken by a CCD camera at 6 frames per second during application of an electric field at $310^{\circ} \mathrm{C}$. Representative still images at selected elapsed times are displayed (Fig. 1c). It is noteworthy that the leading edge of the dark phase that forms propagates in a " $U$ " shape with a minimum near the centerline, indicating an enhancement of the ionic conduction at the edges of the channel. The oxygen-vacancy concentration in the as-grown state is as high as two vacancies $/ 1 \mathrm{~nm}^{3}$. A single oxygen vacancy is known to produce local lattice deformation that decays with a relaxation length of a few unit cells ${ }^{28}$. This high-density defect solution is also likely to experience an elastic interaction between two adjacent oxygen vacancies that are only 1-2 unit cells apart ${ }^{29,30}$. This interaction is likely the origin of the viscous flow at the macroscopic level, creating the U-shaped front with a curvature of ten microns that is reminiscent of capillary motion (Supplementary Fig. 2).

By analyzing the real-time video, we constructed a color-contrast distribution along the centerline as a function of elapsed time after the start of the electroforming process (Fig. 1d). The front of the dark phase moves to the left because the constant external voltage is applied only across the yellowish insulating region that is being shortened; thus, the effective electric-field strength 
$\mathbf{a}$

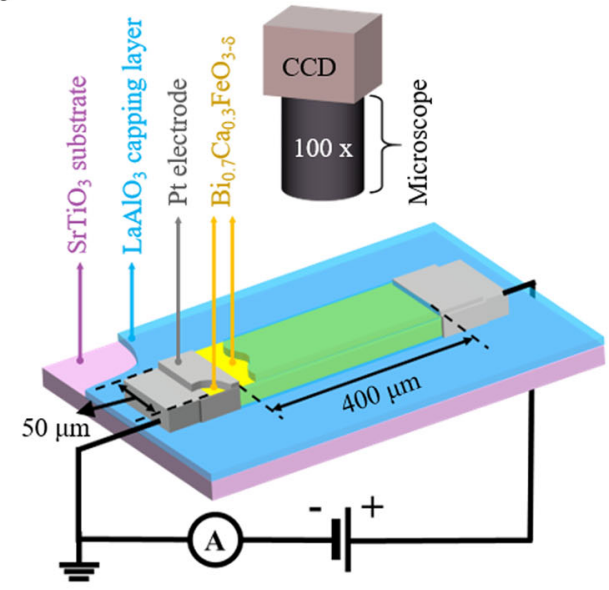

b

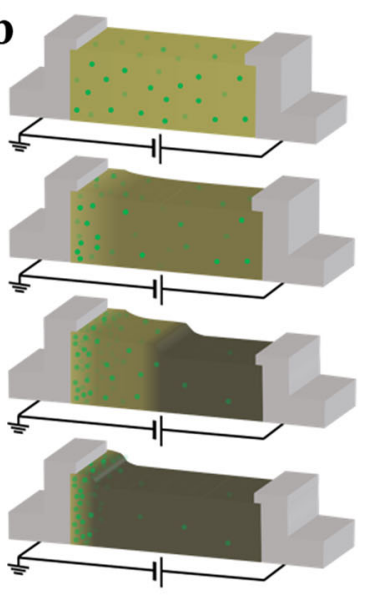

- Oxygen vacancy c
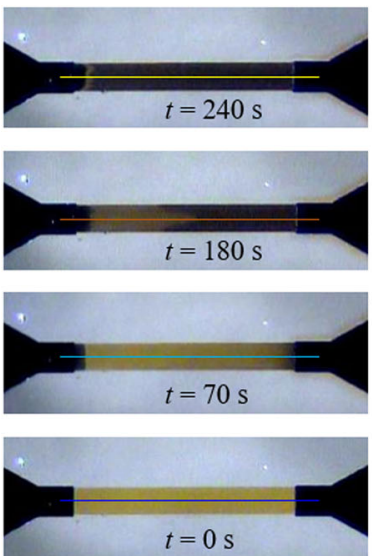

d

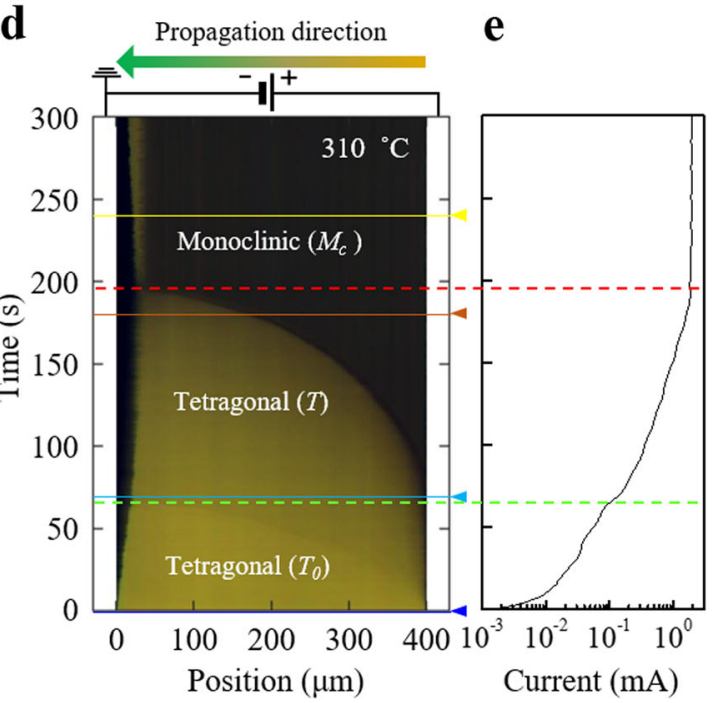

Fig. 1 Real-time visualization of the electroforming process in BCFO film. a Schematic of a BCFO channel protected by a capping layer between a pair of coplanar electrodes with a $400 \mu \mathrm{m}$ gap. Real-time current through the channel and optical microscope video are simultaneously recorded while an electric bias $+25 \mathrm{~V}$ is applied. $\mathbf{b}$ This schematic describes homogeneously distributed oxygen vacancies in the as-grown state that migrate toward the negatively biased electrode, thereby creating a dark-colored formed phase. The front of the phase propagates to the left at a positive bias. c Optical microscope images at elapsed time $t=0 \mathrm{~s}$ (as-grown state), $70 \mathrm{~s}$ (intermediate state), $180 \mathrm{~s}$ (expansion of the formed phase) and $240 \mathrm{~s}$ (completion of a forward-formed state) after the bias is turned on at $310^{\circ} \mathrm{C}$. $\mathbf{d}$ Real-time visualization of the optical contrast along the centerline of the channel during electrical forward forming. The as-grown yellow phase (tetragonal: $T_{0}$ ) turns into an intermediate dark-yellow phase (tetragonal: $T$ ) and finally converts into a dark formed phase (monoclinic: $M_{C}$ ). Color lines and marks correspond to the centerlines of still images in c. e In-situ electrical current monitoring during forming. Curvature of the logarithmic current changes with the $T_{0}$ to $T$ transition (green-dashed line). The current is saturated after completion of the electrical forming process (red-dashed line)

increases with time. In the end, most of the oxygen vacancies accumulate in the narrow $(\sim 10 \%$ of the total channel length) region near the negative bias electrode. No variation in the optical contrast is observed after $190 \mathrm{~s}$, indicating that ionic migration has reached a saturated state. This is consistent with the real-time monitoring of the current in the device, which is also saturated after that time period (Fig. 1e). The BCFO film grown on a $\mathrm{DyScO}_{3}$ substrate also exhibits a dark phase in the absence of oxygen vacancies. This dark phase is not caused by the change in the substrate characteristics but by the change in the BCFO film (Supplementary Fig. 3). Contrary to the dark color in the heavily hole-doped case obtained due to the reduction of oxygen vacancies, the oxygen-vacancyrich phase is semitransparent, i.e., similar to the as-grown state, regardless of the increased oxygen-vacancy concentration. The strong dark color observed near the left electrode after saturation is presumably because the accumulated oxygen vacancies are partially absorbed by the $\mathrm{SrTiO}_{3}$ substrate underneath ${ }^{31,32}$. A careful look at the 
time-position diagram of the optical contrast indicates the existence of two distinct transitions among the as-grown yellow, intermediate dark-yellow, and final dark phases. The phase transition from the as-grown yellow phase to the intermediate dark-yellow phase is complete at $\sim 70 \mathrm{~s}$ with a change in the derivative in the real-time monitoring current, and the transition to the final dark phase is complete at $\sim 190 \mathrm{~s}$ with saturation of the monitoring current.

\section{Evolution of the crystal structure}

For a better understanding of the emergent phases, we performed structural analyses of the various states/phases. The electroformed state can be similarly established in a large-area device $\left(4 \times 1 \mathrm{~mm}^{2}\right)$ by application of $25 \mathrm{~V}$ at $250^{\circ} \mathrm{C}$ for $5 \mathrm{~h}$ (Fig. 2a). The $2 \theta-\omega \mathrm{X}$-ray diffraction scans of the 003-diffraction condition were performed in a spatially resolved manner using a focused X-ray beam while scanning the interelectrode area. According to the position-dependent diffraction results (Fig. 2b), the $c$-axis lattice parameter across the capacitor considerably contracted, confirming the spatial uniformity of the resultant phase after completion of electroforming. One exception is the region near the negative electrode. In this region, even with a very high concentration of accumulated oxygen vacancies, the lattice parameter is comparable with that of the as-grown state. The excessive oxygen vacancies in the region are likely not fully ionized. We note that the $n$-type doped region is electronically insulating and optically transparent due to electron localization near the excessive donors ${ }^{33}$. In addition, the $\mathrm{SrTiO}_{3}$ substrate serves as a sink and effectively absorbs defects when they become too dense. We also carried out reciprocal space mapping studies to clarify the crystal structure of the pseudocubic unit cells for the electroformed and as-grown states (Supplementary Fig. 4). The as-grown phase of BCFO at $x=0.3$ has a tetragonal structure without any noticeable splitting in the asymmetric 103and 113-diffraction peaks and exhibits superlattice reflections along the out-of-plane direction with a period of 5 unit cells, which originates from the oxygen-vacancy superstructure. On the other hand, the electroformed phase exhibits a $M_{C}$-type monoclinic structure with a monoclinic tilt angle of $1.21^{\circ}$, and no superstructure is detected.

To gain further insights into the real-time structural evolution, we employed time-resolved X-ray diffraction along the $L$ axis about the 002-diffraction peak while monitoring the channel current under the same electroforming conditions for the abovementioned large cell (Fig. 2c-f). Despite requiring longer times due to the larger dimensions of the cell, the real-time measurement results reproduce the two anomalies observed in the timeand position-dependent color-contrast diagram (Fig. 1d).
During the establishment of the intermediate phase, the oxygen vacancies have partially migrated, causing a slight contraction of the lattice; however, periodically ordered oxygen vacancies are sustained until the system undergoes a first-order structural transition into the final electroformed state. This isosymmetric transition is continuous in terms of the crystal structure and the relative strength of the superlattice peaks, although the optical contrast is similar but distinguishable. Such a small lattice contraction agrees with the general trend that a reduction in oxygen vacancies results in a lattice contraction by increasing cation-anion interactions ${ }^{34}$. The initiation of the electroformed monoclinic state is marked by the appearance of a shoulder on the right-hand side of the $\mathrm{SrTiO}_{3}$ substrate peak and a corresponding reduction in the intensity of the superlattice peak due to ordered oxygen vacancies. Once the electroformed state is fully established across the channel, the differential current value approaches the maximum, and the conductivity of the BCFO film begins to saturate. After the external voltage is turned off, the formation slowly relaxes, and the asgrown state is restored after maintaining the system at the same temperature for $\sim 3 \mathrm{~h}$. This relaxation to the asgrown state is the result of oxygen reacting through the capping layer; thus, the relaxation time strongly depends on the thickness of the capping layer. We note that a relaxation time of several hours with a thickness $\sim 10 \mathrm{~nm}$ is long enough to provide a quasi-closed system for dynamics experiments through a $400-\mu \mathrm{m}$-channel device. Similar tests with different capping layers $(6$ or $8 \mathrm{~nm}$ thickness) yield almost the same results as the $10-\mathrm{nm}$ thick measurements, but a channel with a thinner $4 \mathrm{~nm}$ capping layer results in a deviation from the other results (Supplementary Fig. 5).

\section{Quantification of kinematic and thermodynamic properties}

On these grounds, we next examined the thermodynamic properties of ionic transport. Time- and positiondependent optical-contrast images were systematically obtained at selected temperatures in the range from 250 to $390^{\circ} \mathrm{C}$ at intervals of $10^{\circ} \mathrm{C}$ (Fig. 3). As expected, the average electromigration speed increases with temperature. The front of the dark-colored phase, i.e., the boundary between the $M_{C}$ and $T$ phases, has a curved shape attributable to acceleration; however, the curvature of the boundary between the $T_{O}$ and $T$ phases is relatively even, indicating that the voltage is applied across the intermediate $T$ phase as well as the as-grown phase. These electronic conduction properties can also be recognized in the channel current (Fig. 4a, b). The initial current quickly increases by three orders of magnitude within the temperature range, indicating that the as-grown phase is highly insulating. In contrast, the saturated current after completion of electroforming is almost independent of 
a
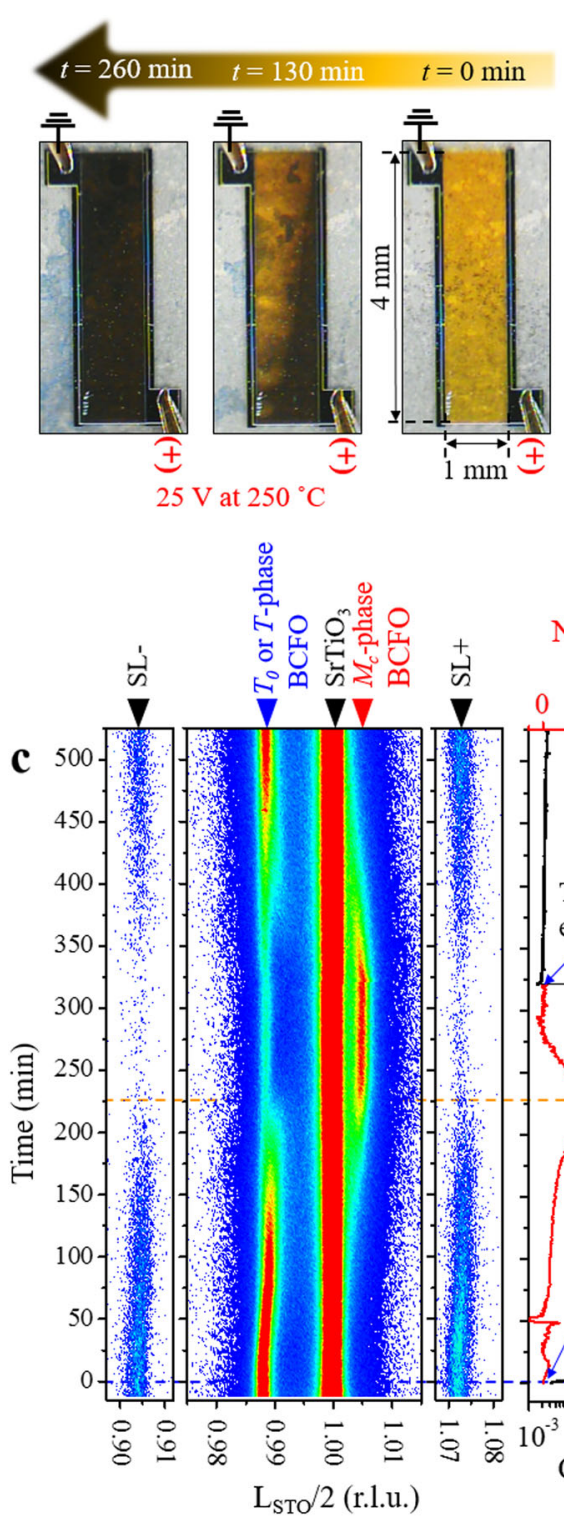

b

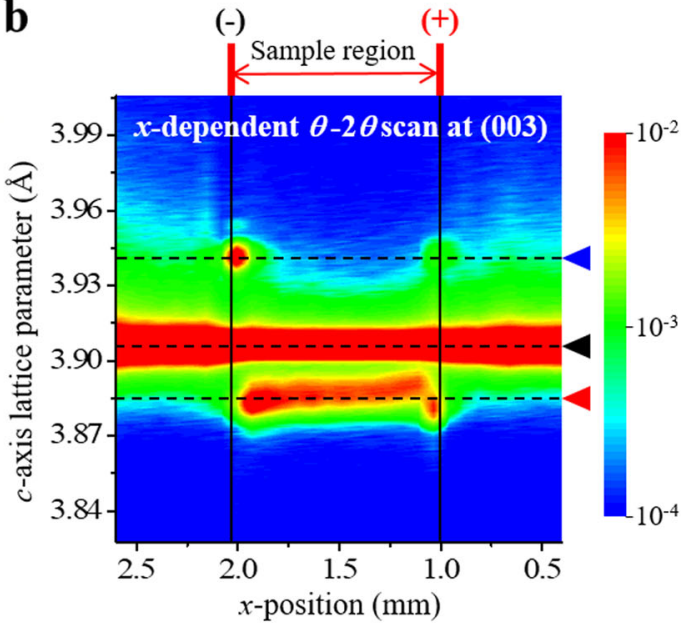




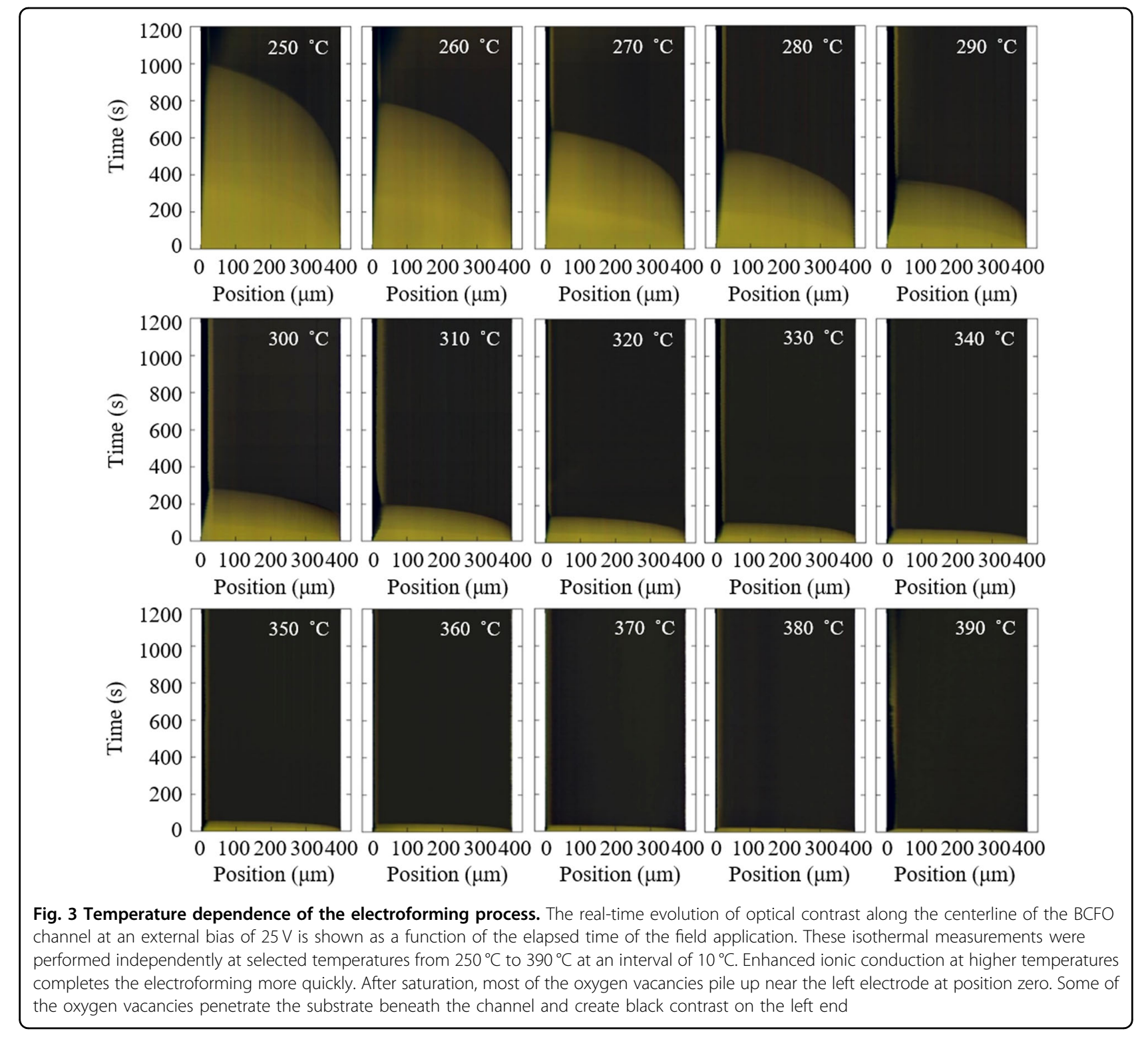

quantitative analysis, the position of the front of the conducting $M_{C}$ phase was extracted from each still image of the video and plotted as a function of elapsed time (Fig. 4c). To analyze the curved trajectories, we introduce a simplified model with the reasonable assumption that all the external voltage $(25 \mathrm{~V})$ is applied across the yellowish insulating region with a length $L-z$, where $L$ is the given channel length $(400 \mu \mathrm{m})$ and $z$ is the length of the conducting region, i.e., the instantaneous position of the front from the right-hand side electrode (Fig. 4d). Thus, the effective electric field $(E)$ in the vacancy-rich region can be written as $V /(L-z)$, and the moving velocity of the front $(d z / d t)$ is comparable to the drift velocity $(u)$ of the vacancies in the electric field. Given that the resistivity is defined as $\rho=E / n q u$, where $n$ is the oxygen-vacancy concentration and $q$ is the ionic charge, we obtain an equation of motion to describe the trajectory of the front movement.

$$
n q \rho(T) \frac{d z}{d t}=\frac{V}{L-z} .
$$

This differential equation can be exactly solved, and the solution can be written as

$$
z(t)=L-\sqrt{L^{2}-\frac{2 V}{n q \rho(T)}\left(t-t_{0}\right)} .
$$

We fit the curve to the experimental data to determine the two unknown parameters: ionic mobility $\mu(T)=(n q \rho$ $(T))^{-1}$ and time offset $t_{0}$. The fit well matches each 

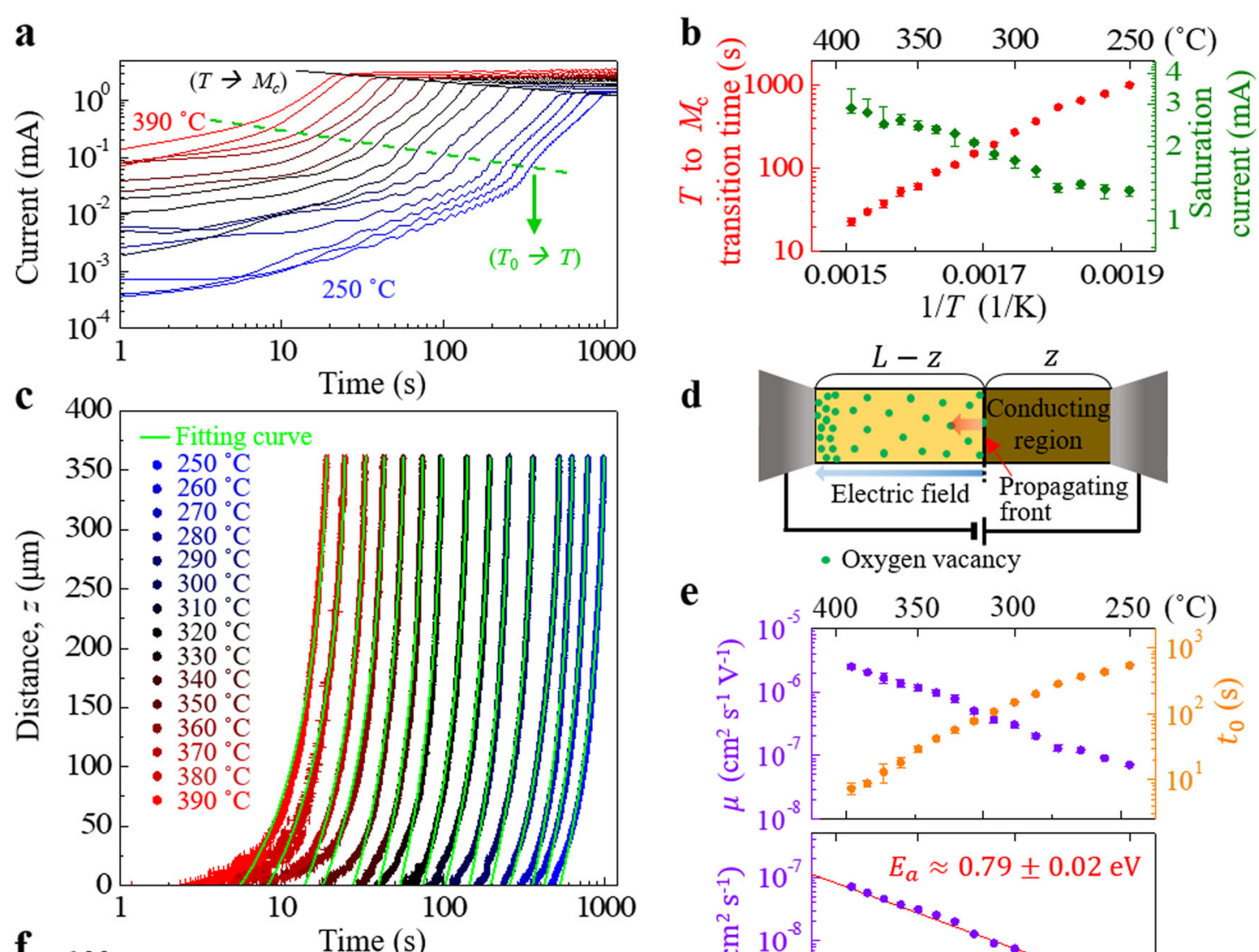

d

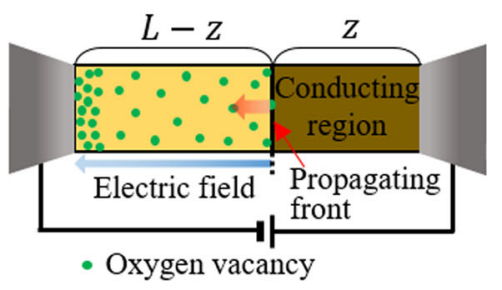

e
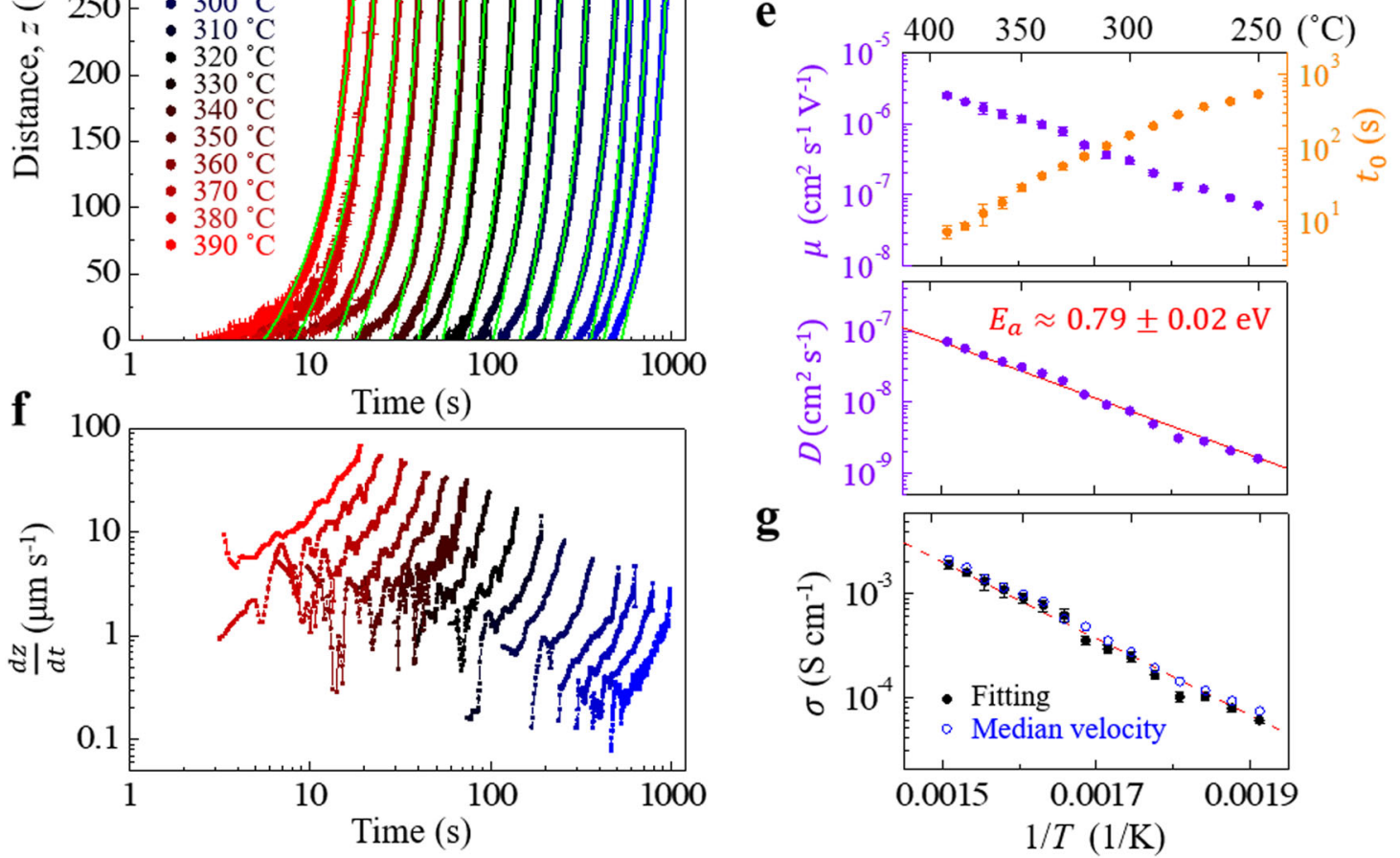

g

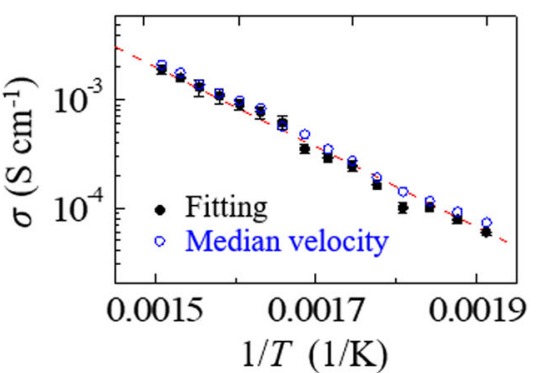

Fig. 4 Quantitative analysis of the electroforming process to determine the dynamic characteristics associated with oxygen-vacancy migration in BCFO. a The real-time monitoring current acquired at a bias of $25 \mathrm{~V}$ while varying the temperature from $250^{\circ} \mathrm{C}$ to $390^{\circ} \mathrm{C}$. A greendashed guideline indicates the transition time from the as-grown phase $\left(T_{0}\right)$ to the intermediate phase $(T)$. The black line represents the time to fulfill the $T$ to $M_{c}$ transition. $\mathbf{b}$ Transition time and saturation current for the $T$ to $M_{c}$ transition. $\mathbf{c}$ The position of the boundary between the $T$ and $M_{c}$ phases is plotted as a function of elapsed time. The error bar is defined as the full-width-at-half-maximum of differential color change. The green lines represent the fitting curves that match the experimental results. $\mathbf{d}$ Simplified model assuming that the applied bias is only across the left region of the front. e The fitting parameters, i.e., ionic mobility and time offset are determined by fitting the data in $\mathbf{c}$ to the model addressed in the main text. Diffusivity can be derived from mobility. $\mathbf{f}$ Instantaneous velocity of the front propagation of the $M_{c}$ phase obtained by differentiation of the experimental data in c. g Arrhenius plot for the ionic conductivities

experimental trajectory, which is displayed as a green line (Fig. 4c). We can reliably determine the two fitting parameters and summarize them as a function of inverse temperature (Fig. 4e). Remarkably, the ionic mobility reaches $\sim 2 \times 10^{-6} \mathrm{~cm}^{2} \mathrm{~s}^{-1} \mathrm{~V}^{-1}$ at $390^{\circ} \mathrm{C}$. Ionic diffusivity $(D)$ can be calculated from $\mu(T)$, according to the relation
$D=\mu k_{B} T / q$, where $k_{B}$ is the Boltzmann constant. The linear relationship in the plot of the logarithm of $D$ versus $1 / T$ provides an activation energy of $\sim 0.79 \mathrm{eV}$. The motion of oxygen vacancies in the edge region is slightly faster than the motion in the center region in the analysis here, but the ionic diffusivities of the two regions are 
almost identical (Supplementary Fig. 6). Alternatively, we can directly deduce the drift velocity by differentiating the experimental trajectory with respect to time (Fig. 4f). The maximum speed of the collective ionic migration is 70 $\mu \mathrm{m} \mathrm{s}^{-1}$ at $390^{\circ} \mathrm{C}$, which implies that oxygen vacancies can completely migrate through a $1 \mathrm{~mm}$-thick BCFO electrolyte within $15 \mathrm{~s}$ under an electric field of $5 \mathrm{kV} \mathrm{cm}^{-1}$ at this surprisingly low operation temperature. Provided that a doubly ionized oxygen vacancy $\left(V_{O}^{*}\right)$ carries a charge of $q=2 \times 1.60 \times 10^{-19} \mathrm{C}$ and $n$ is approximately equal to the as-grown state concentration, i.e., $2.49 \times 10^{23} \mathrm{~cm}^{-3}$ at $\delta=$ $0.3 / 2$, the $\mu(T)$ obtained by the fitting can be converted into an ionic conductivity, $\sigma(T)$ (Fig. $4 \mathrm{~g}$ ). The ionic conductivity is also estimated using the median value of the drift velocities obtained by the direct differentiation at $z$ $=L / 2$. These two evaluations show good agreement. In addition, three BCFO samples with different thicknesses of $37 \mathrm{~nm}, 107 \mathrm{~nm}$, and $220 \mathrm{~nm}$ are similarly examined (Supplementary Fig. 7). We find that the ionic conductivity of the BCFO film is nearly independent of the film thickness, indicating that the interfacial effect between the BCFO film and the $\mathrm{SrTiO}_{3}$ substrate is not significant.

The ionic conductivity in the low-temperature regime studied here is considerably higher than that $\left(\sim 10^{-4}\right.$
$\mathrm{S} \mathrm{cm}^{-1}$ at $400^{\circ} \mathrm{C}$ ) of a yttrium-stabilized zirconia (YSZ) single crystal, which is popular in SOFC research ${ }^{35,36}$. Moreover, the BCFO film has a high oxygen-vacancy diffusivity in the range of $10^{-7}-10^{-9} \mathrm{~cm}^{2} \mathrm{~s}^{-1}$ even if the temperature is lower than $400^{\circ} \mathrm{C}$. We note that these values are promising considering that the diffusivities of other oxygen conductors range from $10^{-9}-10^{-6} \mathrm{~cm}^{2} \mathrm{~s}^{-1}$ in the intermediate-temperature regime $\left(500-800^{\circ} \mathrm{C}\right)$ (see Fig. 5; ref. $\left.{ }^{37-41}\right)$. Operation of oxygen-vacancy transport at a significantly lower temperature enables the choices of interconnection and sealing materials to be expanded and enhances the lifetime of transport cells by reducing thermal degradation. Our results are very encouraging and likely to improve because they have been obtained without optimization of changes in the Ca-substitution ratio and substrate selection or using direction control to manipulate the strain state and dimension control.

Next, we discuss the possible origin of the time offset. All trajectories show that a temperature-dependent finite time $\left[t_{0}(T)\right]$ should elapse for the dark conducting phase to nucleate at the positively biased electrode. If no oxygen vacancies are supplied from the outside of the channel, this would occur suddenly as soon as the electric field is applied. We speculate that the delay arises from surface diffusion of additional oxygen vacancies from the extra

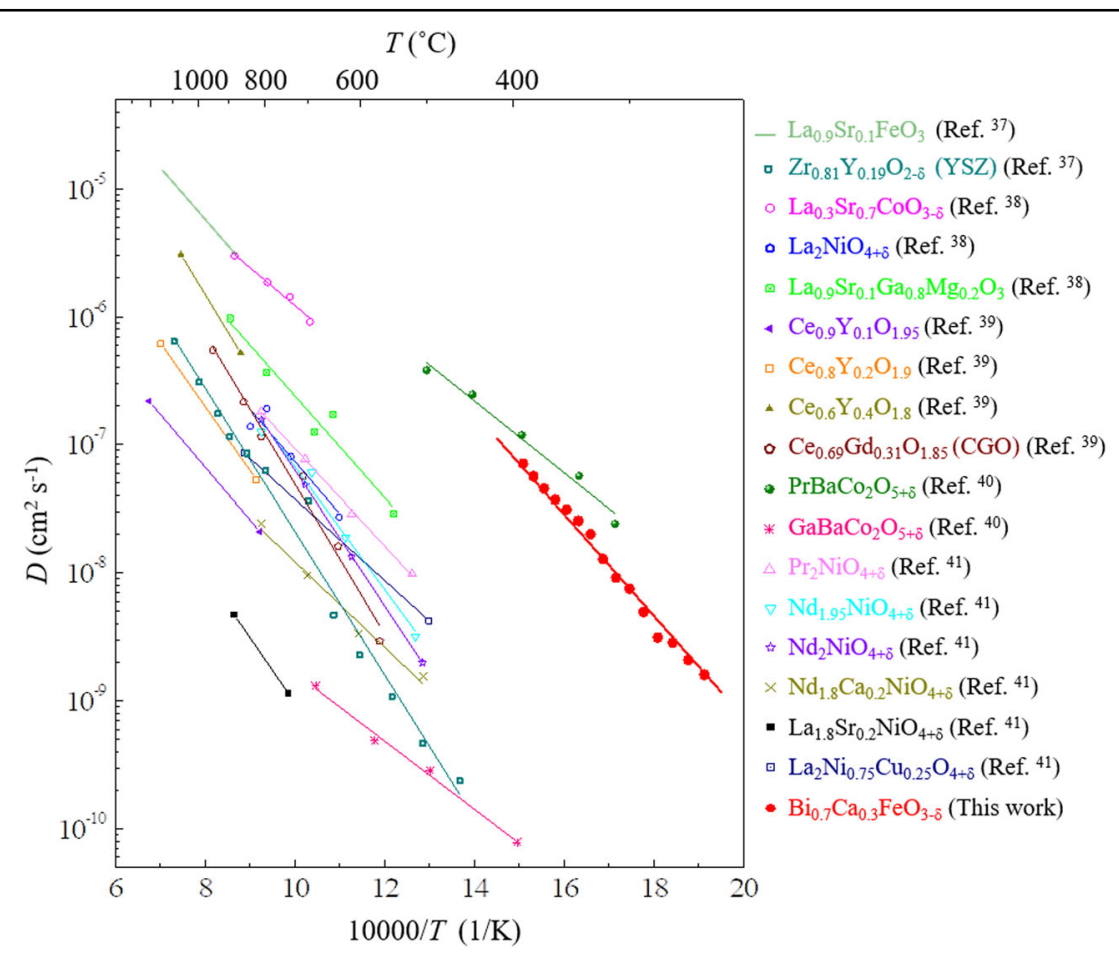

Fig. 5 Comparison of the oxygen diffusivities of various oxide materials and BCFO. The diffusivity of oxygen in the BCFO film in this work is displayed as the red solid circles. Diffusivities of other oxide materials have been determined by the oxygen isotope exchange depth profile method. The data of other oxide materials are adapted from ref. ${ }^{37-41}$ 
channel $(\sim 20 \mu \mathrm{m}$ in length) placed underneath the electrode to provide electric contact. The diffusion length $\left(\sqrt{D t_{0}}\right)$ determined by Fick's second law (Fig. 4e) is on the order of $\sim 10 \mu \mathrm{m}$, which is comparable with the extra channel length. We also cannot exclude the possibility that the Schottky barrier at the interface reduces the effective electric field therein until the barrier is lowered by a defect-induced change in the band gap ${ }^{42}$. The higher the temperature is, the faster oxygen vacancies can overcome the Schottky barrier and/or complete diffusive migration out of the electrode region ${ }^{43}$.

\section{Reversibility and turbulence}

Finally, we investigated whether the highly accumulated oxygen vacancies near the left electrode after forward electroforming can be restored to a more relaxed form and eventually be transferred to the other electrode by a reverse bias. A negative electric bias causes the oxygenvacancy-rich phase to expand from the left electrode and move to the right (Fig. 6a). A significant amount of oxygen vacancies is likely to be absorbed into the substrate when the vacancies accumulate near the left electrode, as indicated by the black color near the left edge of the channel.
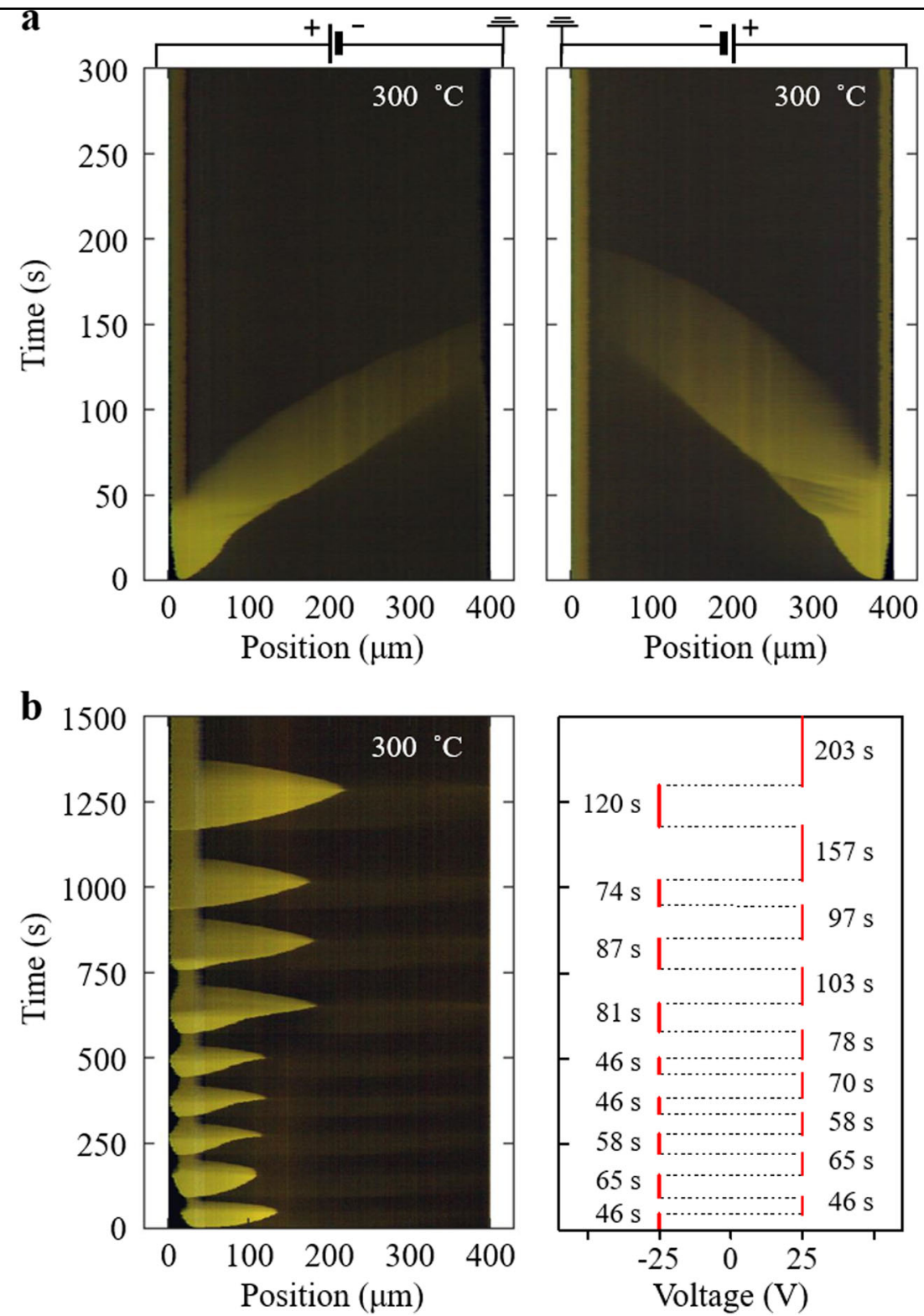

Fig. 6 Reversibility and repeatability of the electroforming. a (Left) Real-time visualization of reverse forming on a completely formed state. (Right) After that, forward forming was repeated. b (Left) Real-time visualization of repeating reverse and forward forming processes. (Right) The profile of the bias applied during the iterative process 
Thus, the number of oxygen vacancies moving backwards is not enough to fill the entire channel; instead, the oxygen-vacancy-rich region extends to a length of $\sim 150$ $\mu \mathrm{m}$. Upon reaching the right electrode, a reverse state is formed, and then, the oxygen-vacancy-rich region moves back to the left under the sequential forward bias. This formed state can be switched repeatedly by the application of two opposite electric fields. Furthermore, switching the polarity of the electric field in the middle of migration creates repetition of the backward and forward motion of the phase boundary (Fig. 6b).

When the accumulated oxygen vacancies near the left electrode are returned to the right by a reverse bias, the oxygen vacancies at the side edges are rapidly released, resulting in an oxygen-vacancy-sparse medium. The relatively low oxygen-vacancy concentration unexpectedly causes turbulent motion, i.e., one of the hallmarks of fluidic dynamics, at the edges (see Supplementary Fig. 8). This fluidic-like motion is likely a result of the large number of oxygen vacancies that electrostatically and sterically interact in the solid and behave like chargedfluid particles in an electrolyte $e^{44,45}$.

\section{Conclusions}

We found that the spontaneously produced oxygen vacancies in BCFO films are highly mobile, even in lowtemperature regimes. By tracking the visualized electroforming processes, we were able to directly observe the propagation of the boundary between the oxygenvacancy-rich and -poor competing phases through a channel protected by an oxygen barrier and quantitatively determine the kinematic and thermodynamic variables of the ionic motion. The drift velocity of the collective migration was on the order of $100 \mathrm{\mu m} \mathrm{s}^{-1}$, and the oxygen-vacancy diffusivity was quantified as $7 \times$ $10^{-8} \mathrm{~cm}^{2} \mathrm{~s}^{-1}$ at a low temperature of $390^{\circ} \mathrm{C}$. These results provide a promising material for low-temperature oxygen conduction and offer an alternative pathway for the visualization and quantification of defect dynamics.

\section{Methods}

\section{Synthesis of epitaxial BCFO thin films}

The epitaxial $\mathrm{Bi}_{0.7} \mathrm{Ca}_{0.3} \mathrm{FeO}_{3-\delta}$ thin films were grown on a $\mathrm{SrTiO}_{3}$ (001) substrate (CrysTec $\mathrm{GmbH}$ ) using pulsed laser deposition with a $\mathrm{KrF}$ excimer laser $(\lambda=248 \mathrm{~nm})$. A pellet with $10 \%$ bismuth excess was prepared by mixing $\mathrm{Bi}_{2} \mathrm{O}_{3}$ (99.9\%), $\mathrm{CaO}$ (99.95\%), and $\mathrm{Fe}_{2} \mathrm{O}_{3}$ (99.9\%) powders (Sigma-Aldrich) and forming the mixture. The pellet was calcined at $600{ }^{\circ} \mathrm{C}$ for $6 \mathrm{~h}$ under ambient conditions. After calcination, the pellet was crushed, ground, and pressurized to create a 1-inch diameter button-shaped target. Then, it was sintered at $650{ }^{\circ} \mathrm{C}$ for $6.5 \mathrm{~h}$ under ambient conditions. The epitaxial BCFO thin films $(\sim 100 \mathrm{~nm}$ in thickness) were grown at a heater temperature of $650{ }^{\circ} \mathrm{C}$ in an oxygen environment of 0.05 Torr. The laser fluence and repetition rate were set to $\sim 1 \mathrm{~J} \mathrm{~cm}^{-2}$ and $10 \mathrm{~Hz}$, respectively. All the samples were cooled to room temperature at a rate of $10^{\circ} \mathrm{C} \mathrm{min}-1$ at an oxygen pressure of 500 Torr.

\section{Fabrication of BCFO microdevices}

Typical UV-lithography using AZ5214E photoresist (AZ Electronic Materials) was carried out to pattern eight bars $(440 \mu \mathrm{m} \times 50 \mu \mathrm{m})$ on each as-grown BCFO thin film. The patterned samples were dry-etched by $\mathrm{Ar}^{+}$-ion milling using a $2 \mathrm{~cm}$ diameter ion beam source. All the BCFO areas, except for the patterned regions, were etched at a rate of $\sim 1 \mathrm{~nm} / \mathrm{min}$. During the etching process, the sample holder was continuously cooled using a cooling water system to prohibit thermal decomposition and degradation. To minimize the reaction with the atmosphere during the electrical forming experiments, we additionally deposited a $\mathrm{LaAlO}_{3}$ capping layer $(10 \mathrm{~nm}$ thickness) on the whole sample surface over the substrate area as well as the remaining unetched BCFO regions by pulsed laser deposition. The epitaxial capping layer was grown at a temperature of $650{ }^{\circ} \mathrm{C}$ in oxygen gas at 0.05 Torr under a laser fluence and repetition rate of $\sim 1 \mathrm{~J} \mathrm{~cm}^{-2}$ and $2 \mathrm{~Hz}$, respectively. After deposition, the sample was cooled down to room temperature at a rate of $10^{\circ} \mathrm{C} \mathrm{min}-1$ at an oxygen pressure of 500 Torr. The platinum electrodes required for electrical contact with external measurement equipment were patterned by using the abovementioned UV-lithography technique. The spacing between the platinum electrodes was $400 \mu \mathrm{m}$, and the vertical length of the bars was $50 \mu \mathrm{m}$. The $\mathrm{LaAlO}_{3}$ capping layers on the patterned positions were removed by $\mathrm{Ar}^{+}-$ ion milling, and then, platinum was in-situ deposited by DC magnetron sputtering at an argon pressure of $5 \mathrm{mTorr}$ with a power of $25 \mathrm{~W}$ at room temperature to contact the platinum electrodes directly to the surface of BCFO. The large BCFO bars, $4 \mathrm{~mm}$ long and $1 \mathrm{~mm}$ wide, for the $\mathrm{X}$ ray-based structural analysis were also fabricated using the same procedure.

\section{Optical visualization and electrical measurement}

The temperature of the patterned BCFO films was controlled by a custom-made heating stage installed in an optical microscope (HiMax Tech, HNM005), and two electrical probe tips were connected to platinum electrodes to apply an external voltage and measure the electrical current during electroforming. The custommade heating stage was composed of a $\mathrm{Ni}-\mathrm{Cr}$ alloy wire enclosed with an alumina mold. A K-type thermocouple was attached near the heating stage. The BCFO films for the optical measurements were glued on the heating stage 
using a sliver paste for better thermal contact. An external white LED light source was illuminated at $\sim 15^{\circ}$ from the sample surface. The surfaces of the BCFO films were imaged through a $10 \times$ objective lens and a $10 \times$ eyepiece at a magnification of 100 , and the working distance of the objective lens was $34 \mathrm{~mm}$, allowing the probe tips to move sufficiently. Two gold-coated probe tips on positioners (MS Tech) connected the platinum electrodes of the BCFO films to a voltage source (Tektronix, Keithley 230) and a current meter (Tektronix, Keithley 2000). A Color CCD (Veltek, CVC-5220) camera in the optical microscope recorded the electrical forming process at a rate of 6 frames per second. The patterned BCFO films were electrically formed by an external voltage of $25 \mathrm{~V}$ at high temperatures, and the electrical current and video image were simultaneously recorded.

\section{Investigation of oxygen-vacancy trajectories via video imaging analysis}

Each video was converted into frame images using Adobe Premiere Pro CC 2015 software. Each frame had a resolution of $720 \times 480$ pixels, and each pixel had $\mathrm{R}(\mathrm{red})$, G (green), and B (blue) information. A sample area was divided into 9 parts along its width direction, and the color change depending on time was analyzed for each image. To investigate the effect of temperature, the middle region of the sample, which had a width of $\sim 5.6 \mu \mathrm{m}$ and included 5 pixels, was analyzed as a function of temperature. The frame order was arranged by referring to the time of the video, and the average value of the pixels along the width direction represented the color information of the position. Stacking the color information in frame order allowed a profile to be created to observe the color change over time. Since we measured samples in different regions for each temperature, the RGB color information was interpolated to have $R$ values between 0.1 and 0.6 , G values between 0.1 and 0.55 and, B values between 0.1 and 0.15 for comparison. The RGB interpolation reference value was determined from the average RGB value of the initial sample color and the electrically formed sample color. The dark and dark yellow boundaries represent the motion trajectory of the oxygen vacancies. The trajectory information was obtained by applying differentiation with respect to the position along the time axis. The minimum value of differentiation provides information on the trajectory. The full-width-at-half-maximum of the Gaussian peak of the derivative gives the time error information. Similarly, position error information could be obtained from the Gaussian peak of the differential along the position axis. The parameters of the fitting equation for the trajectories were extracted using Origin 8.5 software. The velocity of the oxygen vacancies was calculated from the differential of the trajectory over time.

\section{Spatially resolved X-ray diffraction of the electrically formed BCFO thin film}

The spatially resolved analysis for the out-of-plane lattice parameter of the electrically formed 1-mm-wide BCFO film was performed using $\mathrm{X}$-ray $\theta-2 \theta$ scans as a function of the $X$ position of the sample holder at the beamline (3A-RXS) of the Pohang Light Source at the Pohang Accelerator Laboratory. The BCFO film for synchrotron measurement was 100 -nm thick and was capped by $\mathrm{LaAlO}_{3}$ to prohibit any external effect on the sample composition. The large BCFO device was used for synchrotron-based X-ray diffraction. The application of $25 \mathrm{~V}$ to the fabricated BCFO film transformed the as-grown area into an electrically formed area, and then, we quickly cooled the sample to room temperature for the purpose of maintaining the created electrically formed area. This electrically formed BCFO film was mounted on a sample goniometer equipped with a programmable $X Y$ stage. A Si (111) double-crystal monochromator was utilized to generate an X-ray beam of 10.99 $\mathrm{keV}$, which corresponds to $\lambda=1.128616 \AA$. The incident $\mathrm{X}$-ray beam was cut to a width of $\sim 70 \mu \mathrm{m}$ by a series of incident slits, and the diffracted beam was collected by a scintillator detector via a highly oriented pyrolytic graphite (002) analyzer. We investigated the (003) film peak to make the projected beam size on the sample less than $150 \mu \mathrm{m}$. The $X$ position of the mounted BCFO sample was moved along the width direction in $100-\mu \mathrm{m}$ steps. This measurement was achieved within $3 \mathrm{~h}$. When an electrically formed BCFO film was exposed to the strong synchrotron X-ray beam for a long time, the electrically formed area relaxed due to local heating.

\section{Time-resolved structural analysis of the BCFO thin film}

Reciprocal space maps (RSMs) for the (103) and (113) reflections under ambient conditions were measured for the as-grown, electrically formed, and thermally relaxed states of a BCFO film using a four-circle X-ray diffractometer (PANalytical X'Pert PRO MRD) with $\mathrm{Cu} \mathrm{K}_{\alpha 1}$ radiation. The size of the BCFO sample for the RSM measurement was $4 \mathrm{~mm} \times 1 \mathrm{~mm}$, which was large enough to carry out the diffraction experiments. We performed $\theta$ $2 \theta$ scans from $10^{\circ}$ to $90^{\circ}$ with $1 \mathrm{~s}$ per point at an interval of $0.1^{\circ}$. The (001), (002) and, (003) film peaks and superlattice peaks were observed. The as-grown crystal structure was tetragonal considering that the $Q_{z}$ positions of the (103) and (113) peaks were equal with no splitting. The $Q_{x}$ positions of the film peaks were also observed to be equal to those of the substrate in both RSMs, indicating that the film was fully strained with the substrate. In the case of the electrically formed state, it was observed that the (103) and (113) film peaks were split along the out-of-plane $Q_{z}$ direction. By performing $Q_{z}$ scans of the (103) and (203) peaks, we observed that the peak splitting in the (203) peak was twice that in (103), indicating a 
monoclinic structure. The electrically formed BCFO state was converted to the thermally relaxed $\mathrm{BCFO}$ state by heating the sample at $250^{\circ} \mathrm{C}$ for approximately 1 day. We obtained the (103) and (113) RSMs of the thermally relaxed $\mathrm{BCFO}$ state using the same experimental procedure. In all three states, $Q_{x}$ scans were performed to check the quality of the film.

Time-resolved X-ray diffraction was investigated using a 2D array detector (PIXel ${ }^{3 \mathrm{D}}$ detector) with a hightemperature stage (Anton Paar, DHS-1100). The BCFO film was mounted on the high-temperature stage, and its temperature was raised to $250^{\circ} \mathrm{C}$. An enameled copper wire of $0.1 \mathrm{~mm}$ in diameter was attached to each platinum electrode of the large BCFO film using silver epoxy. Long enameled copper wires were connected to a voltage source (Tektronix, Keithley 230) and a current meter (Tektronix, Keithley 2000) to apply an external voltage and measure the electrical current, respectively. A graphite dome that covered the high-temperature stage stabilized the thermal environment of the sample. One $2 \theta$ scan from $40^{\circ}$ to $52^{\circ}$ took $1 \mathrm{~min}$. Starting with an external voltage of $25 \mathrm{~V}$, a series of $\theta-2 \theta$ scans were sequentially measured as a function of time. The monitoring current was simultaneously measured to infer the state of BCFO at each moment. After approximately $5 \mathrm{~h}$, the external voltage was turned off to allow the electrically formed state to return to the as-grown state while maintaining the temperature. The position, width, and area of each peak were inspected as a function of time using Gaussian fitting.

\section{Acknowledgements}

This work was supported by National Research Foundation (NRF) Grants funded by the Korean Government via the Creative Research Center for Lattice Defectronics (NRF-2017R1A3B1023686) and the Center for Quantum Coherence in Condensed Matter (2016R1A5A1008184). X-ray studies were carried out at the Pohang Accelerator Laboratory. R. G. acknowledges support from the National Science Foundation under grant OISE-1545907. L. W. M. acknowledges support from the U.S. Department of Energy, Office of Science, Office of Basic Energy Sciences, Materials Sciences and Engineering Division under Contract no. DE-AC02-05-CH11231: Materials Project program KC23MP for development of novel functional materials.

\section{Author details}

${ }^{1}$ Department of Physics, Korea Advanced Institute of Science and Technology (KAIST), Yuseong-gu, Daejeon 34141, Republic of Korea. ${ }^{2}$ Center for Lattice Defectronics, KAIST, Yuseong-gu, Daejeon 34141, Republic of Korea. ${ }^{3}$ Department of Materials Science and Engineering, University of California, Berkeley, CA 94720, USA. ${ }^{4}$ Pohang Accelerator Laboratory, POSTECH, Pohang, Gyeongbuk 37673, Republic of Korea. ${ }^{5}$ Materials Sciences Division, Lawrence Berkeley National Laboratory, Berkeley, CA 94720, USA. ${ }^{6}$ Department of Physics, University of California, Berkeley, CA 94720, USA. ${ }^{7}$ KAIST Institute for the NanoCentury, KAIST, Yuseong-gu, Daejeon 34141, Republic of Korea

\section{Conflict of interest}

The authors declare that they have no conflict of interest.

\section{Publisher's note}

Springer Nature remains neutral with regard to jurisdictional claims in published maps and institutional affiliations.
Supplementary information is available for this paper at https://doi.org/ 10.1038/s41427-018-0087-5.

Received: 15 May 2018 Revised: 9 August 2018 Accepted: 17 August 2018. Published online: 27 September 2018

\section{References}

1. Cava, R. J. et al. Oxygen stoichiometry, superconductivity and normal-state properties of $\mathrm{YBa}_{2} \mathrm{Cu}_{3} \mathrm{O}_{7-\delta}$. Nature 329, 423-425 (1987).

2. Ritter, $C$. et al. Influence of oxygen content on the structural, magnetotransport, and magnetic properties of $\mathrm{LaMnO}_{3+\delta}$. Phys. Rev. B 56, 8902 (1997).

3. Qiao, Q. et al. Direct observation of oxygen-vacancy-enhanced polarization in a $\mathrm{SrTiO}_{3}$-buffered ferroelectric $\mathrm{BaTiO}_{3}$ film on GaAs. Appl. Phys. Lett. 107, 201604 (2015).

4. Park, C. H. \& Chadi, D. J. Microscopic study of oxygen-vacancy defects in ferroelectric perovskites. Phys. Rev. B 57, R13961 (1998).

5. Waser, R. \& Aono, M. Nanoionics-based resistive switching memories. Nat. Mater. 6, 833-840 (2007).

6. Kharton, V. V., Marques, F. M. B. \& Atkinson, A. Transport properties of solid oxide electrolyte ceramics: a brief review. Solid State Ion. 174, 135-149 (2004).

7. Mefford, J. T. et al. Anion charge storage through oxygen intercalation in $\mathrm{LaMnO}_{3}$ perovskite pseudocapacitor electrodes. Nat. Mater. 13, 726-732 (2014).

8. Ramamoorthy, R., Dutta, P. K. \& Akbar, S. A. Oxygen sensors: materials, methods, designs and applications. J. Mater. Sci. 38, 4271-4282 (2003).

9. Joshua, Y. J. et al. Memrisitive switching mechanism for metal/oxide/metal nanodevicces. Nat. Nanotechnol. 3, 429-433 (2008).

10. Jo, S. H. et al. Nanoscale memristor device as synapse in neuromorphic systems. Nano. Lett. 10, 1297-1301 (2010).

11. Cherry, M., Islam, M. S. \& Catlow, C. R. A. Oxygen-ion migration in perovskitetype oxides. J. Solid State Chem. 118, 125132 (1995).

12. Khan, M. S., Islam, M. S. \& Bates, D. R. Dopant substitution and ion migration in the $\mathrm{LaGaO}_{3}$-based oxygen ion conductor. J. Phys. Chem. B 102, 3099-3104 (1998).

13. Zhou, Y. et al. Strongly correlated perovskite fuel cells. Nature 534, 231-234 (2016).

14. Marthinsen, A., Faber, C., Aschauer, U., Spaldin, N. A. \& Selbach, S. M. Coupling and competition between ferroelectricity, magnetism, strain, and oxygen vacancies in $\mathrm{AMnO}_{3}$ perovskites. MRS Commun. 6, 182-191 (2016).

15. Chu, K. et al. Enhancement of the anisotropic photocurrent in ferroelectric oxides by strain gradients. Nat. Nanotech. 10, 972-979 (2015).

16. Mueller, D. N., Machala, M. L., Bluhm, H. \& Chueh, W. C. Redox activity of surface oxygen anions in oxygen-deficient perovskite oxides during electrochemical reactions. Nat. Commun. 6, 6097 (2015).

17. Yang, C.-H. et al. Electric modulation of conduction in multiferroic Ca-doped $\mathrm{BiFeO}_{3}$ films. Nat. Mater. 8, 485-493 (2009).

18. Masó, N. \& West, A. R. Electrical properties of Ca-doped $\mathrm{BiFeO}_{3}$ ceramics: from p-type semiconduction to oxide-ion conduction. Chem. Mater. 24, 2127-2132 (2012).

19. Lim, J. S. et al. Electric-field-induced insulator to Coulomb glass transition via oxygen-vacancy migration in Ca-doped $\mathrm{BiFeO}_{3}$. Phys. Rev. B 94, 035123 (2016).

20. Seidel, J. et al. Prominent electrochromism through vacancy-order melting in a complex oxide. Nat. Commun. 3, 799 (2012)

21. Infante, I. C. et al. Bridging multiferroic phase transitions by expitaxial strain in $\mathrm{BiFeO}_{3}$. Phys. Rev. Lett. 105, 057601 (2010).

22. Lee, D. et al. Emergence of room-temperature ferroelectricity at reduced dimensions. Science 349, 1314-1317 (2015).

23. Li, M. et al. A family of oxide ion conductors based on the ferroelectric perovskite $\mathrm{Na}_{0.5} \mathrm{Bi}_{0.5} \mathrm{TiO}_{3}$. Nat. Mater. 13, 31-35 (2014).

24. Aidhy, D. S., Sinnott, S. B., Wachsman, E. D. \& Phillpot, S. R. Effect of ionic polarizability on oxygen diffusion in $\delta-\mathrm{Bi}_{2} \mathrm{O}_{3}$ from atomistic simulation. Ionics 16, 297-303 (2010).

25. Lobaskin, V., Burkhard, D. \& Holm, C. Electrophoretic mobility of a charged colloidal particle: a computer simulation study. J. Phys.: Condes. Matter $\mathbf{1 6}$ S4063-S4073 (2004)

26. Sayre, J. D., Delaney, K. T. \& Spaldin, N. A. Interplay between strain and oxygen vacancies in lanthanum aluminate. Preprint at https://arxiv.org/1202.1431 (2012). 
27. Curnan, M. T. \& Kitchin, J. R. Effects of concentration, crystal structure, magnetism, and electronic structure method on first-principles oxygen vacancy formation energy trends in perovskites. J. Phys. Chem. C. 118, 28776-28790 (2014).

28. Kim, Y.-M. et al. Probing oxygen vacancy concentration and homogeneity in solid-oxide fuel-cell cathode materials on the subunit-cell level. Nat. Mater. 11 888-894 (2012).

29. Yamanouchi, H. \& Fontaine, D. D. Elastic interaction of defect clusters with arbitrary strain fields in an anisotropic continuum. Acta Metall. 27, 763-776 (1979).

30. Shi, Y., Bork, A. H., Schweiger, S. \& Rupp, J. L. M. The effect of mechanical twisting on oxygen ionic transport in solid-state energy conversion membranes. Nat. Mater. 14, 721-727 (2015).

31. Tan, $\mathrm{H}$. et al. Oxygen vacancy enhanced photocatalytic activity of perovskite $\mathrm{SrTiO}_{3}$. ACS Appl. Mater. Interfaces 6, 19184-19190 (2014).

32. Jalan, B., Engel-Herbert, R., Mates, E. T. \& Stemmer, S. Effects of hydrogen anneals on oxygen deficient $\mathrm{SrTiO}_{3-x}$ single crystals. Appl. Phys. Lett. 93, 052907 (2008).

33. Bharathi, K. K. et al. Detection of electrically formed photosensitive area in Cadoped $\mathrm{BiFeO}_{3}$ thin films. Appl. Phys. Lett. 102, 012908 (2013).

34. Ullmann, H. \& Trofimenko, N. Estimation of effective ionic radii in highly defective perovskite-type oxides from experimental data. J. Alloy. Compd. 316, 153-158 (2001).
35. Hui, S. R. et al. A brief review of the ionic conductivity enhancement for selected oxide electrolytes. J. Power Sources 172, 493-502 (2007).

36. Wachsman, E. D. \& Lee, K. T. Lowering the temperature of solid oxide fuel cells. Science 334, 935-939 (2011).

37. Kilner, J. A. Fast oxygen transport in acceptor doped oxides. Solid State lon. 129, 13-23 (2000).

38. Skinner, S. J. \& Kilner, J. A. Oxygen diffusion and surface exchange in $\mathrm{La}_{2-x} \mathrm{Sr}_{x} \mathrm{NiO}_{4+\delta}$. Solid State Ion. 135, 709-712 (2000).

39. Rushton, M. J. D. et al. Effect pf strain on the oxygen diffusion in yttria and gadolinia co-doped ceria. Solid State Ion. 230, 37-42 (2013).

40. Parfitt, D., Chroneos, A., Tarancón, A. \& Kilner, J. A. Oxygen ion diffusion in cation ordered/disordered $\mathrm{GaBaCO}_{2} \mathrm{O}_{5+\delta}$. J. Mater. Chem. 21, 2183-2186 (2011).

41. Boehm, E. et al. Oxygen diffusion and transport properties in nonstoichiometric $\mathrm{Ln}_{2-x} \mathrm{NiO}_{4+\delta}$ oxides. Solid State lon. 176, 2717-2725 (2005).

42. Spicer, W. E. et al. Unified mechanism for Schottky barrier formation and III-V oxide interface states. Phys. Rev. Lett. 44, 420-423 (1980).

43. Tung, R. T. Electron transport at metal-semiconductor interfaces: general theory. Phys. Rev. B 45, 13509-12523 (1992).

44. Anderson, J. L. Colloid transport by interfacial forces. Ann. Rev. Fluid Mech. 21, 61-99 (1989).

45. Poulin, P., Holger, S., Lubensky, T. C. \& Weitz, D. A. Novel colloidal interactions in anisotropic fluids. Science 275, 1770-1773 (1997). 